\title{
Vibrotactile Stimulation as an Instructor for Mimicry-Based Physical Exercise
}

\author{
Jani Lylykangas, ${ }^{1}$ Jani Heikkinen, ${ }^{2}$ Veikko Surakka, ${ }^{1}$ Roope Raisamo, \\ Kalle Myllymaa, ${ }^{1}$ and Arvo Laitinen ${ }^{3}$ \\ ${ }^{1}$ Tampere Unit for Computer-Human Interaction (TAUCHI), School of Information Sciences, University of Tampere, \\ Kanslerinrinne 1, 33014 Tampere, Finland \\ ${ }^{2}$ The Unit of Human-Centered Technology (IHTE), Department of Pervasive Computing, Tampere University of Technology, \\ P.O. Box 553, 33101 Tampere, Finland \\ ${ }^{3}$ Audio Riders, Ltd., Envallinkuja 4B, 01900 Nurmijärvi, Finland
}

Correspondence should be addressed to Jani Lylykangas; jani.lylykangas@uta.fi

Received 18 June 2015; Revised 11 September 2015; Accepted 29 September 2015

Academic Editor: Marco Mamei

Copyright (C) 2015 Jani Lylykangas et al. This is an open access article distributed under the Creative Commons Attribution License, which permits unrestricted use, distribution, and reproduction in any medium, provided the original work is properly cited.

The present aim was to investigate functionality of vibrotactile stimulation in mimicry-based behavioral regulation during physical exercise. Vibrotactile stimuli communicated instructions from an instructor to an exerciser to perform lower extremity movements. A wireless prototype was tested first in controlled laboratory conditions (Study 1) and was followed by a user study (Study 2) that was conducted in a group exercise situation for elderly participants with a new version of the system with improved construction and extended functionality. The results of Study 1 showed that vibrotactile instructions were successful in both supplementing and substituting visual knee lift instructions. Vibrotactile stimuli were accurately recognized, and exercise with the device received affirmative ratings. Interestingly, tactile stimulation appeared to stabilize acceleration magnitude of the knee lifts in comparison to visual instructions. In Study 2 it was found that user experience of the system was mainly positive by both the exercisers and their instructors. For example, exercise with vibrotactile instructions was experienced as more motivating than conventional exercise session. Together the results indicate that tactile instructions could increase possibilities for people having difficulties in following visual and auditory instructions to take part in mimicry-based group training. Both studies also revealed development areas that were primarily related to a slight delay in triggering the vibrotactile stimulation.

\section{Introduction}

Structured physical group exercise is typically based on seeing and listening to instructions of a trainer and then modeling them by trainees who try to do the same movements in synchrony with the trainer. There are many groups, however, like aged and disabled people who cannot benefit or get the full utility from such activities if their sensory or cognitive impairments inhibit them from following visual and auditory instructions. Utilizing the sense of touch as an information channel could partly replace the requirements of the full functionality of visual and auditory perception (see [1] for a review). Following this, touch mediated instructions could increase the achievability of group exercise for special groups and it could actually complement the exercise even in the case of fully functional sensory systems.

Motor response to a touch sensation is an innate trait for human beings, and the sense of touch has some advantages over the other senses [2]. For example, reaction times (RTs) to tactile stimuli have been found to be faster than RTs to visual stimuli (e.g., [3]). As compared to auditory RTs, tactile RTs have been found to be less affected by distractions such as a concurrent secondary task (e.g., [4]). This is probably because, in contrast to visual and auditory events, tactile events are in direct physical contact with the organism and thus evoke more readily the interest of an organism [5]. Mechanisms to react to tactile stimulation are among the first ones to develop as evidenced by the palmar grasp reflex 
of fetuses [6]. Furthermore, the importance of the sense of touch has been shown by, for example, remaining of motor responses to tactile stimulation, while the patients are unresponsive to other types of stimulations like visual and auditory stimulations [7].

Relatively recent experimental research has shown that technology-mediated vibrotactile stimulation (i.e., cutaneous stimulation produced by a vibration actuator) can be successfully used in assisting physical rehabilitation and exercise by means of sensory substitution and augmentation. Wearable vibrotactile feedback systems have proven to be usable, for example, in gait retraining [8], body balance control [9], rowing [10], snowboarding [11], and even swimming [12]. There is also evidence that appropriately designed vibrotactile signals can be intuitively associated with cues suggesting, for example, to accelerate and decelerate the speed of movement [13]. The evidence on intuitive associations between tactile stimulation and human behavior suggests that tactile stimulation might also function as a substitute for another person's visual demonstration of bodily movements. Lee and his colleagues $[14,15]$ have empirically tested body balance training with tactile information in the mimicry frame of reference, but otherwise using tactile stimulation in providing interpersonal, mimicry-based behavioral regulation information seems to be still largely unstudied. In a preliminary study by Lee and Sienko [14] the task was to replicate prerecorded trunk tilt movements consisting of a $20^{\circ}$ anterior tilt followed by a $6 \mathrm{~s}$ long static hold and a $20^{\circ}$ posterior trunk tilt back to the upright starting position. The tilt movements were performed at a rate of about $1.12^{\circ} / \mathrm{s}$. The instructions were either visual, tactile, or combined visualtactile cues. Visual instruction was provided with a side-view illustration of a virtual avatar representing the prerecorded example movements of an instructor. Tactile instructions were provided with two separate vibration actuators placed on trunk midline in the navel and back indicating the anterior and posterior tilts, respectively. End of the vibration communicated the attainment of the desired tilt angle. The results showed that the example tilts were mimicked significantly more accurately and faster with combined visual-tactile and tactile instructions as compared to visual instructions. In a follow-up study [15] the speed of the example movement was varied, and this time only tactile instructions were provided. The results showed that slow (approximately $1.12^{\circ} / \mathrm{s}$ ) example movements were mimicked more accurately as compared to medium (approximately $2.0 \%$ s) and fast (approximately $4.0^{\circ} / \mathrm{s}$ ) example movements. Together the results of Lee and colleagues $[14,15]$ indicate a serious potential of using tactile instructions to mimic physical movement of another person in eyes- and hands-free fashion. However, more research is needed to assess the functionality of the concept in other types of mimicry-based physical training.

In contrast to earlier work, our aim was to broaden the scope from personal (i.e., one-to-one) balance training towards faster-paced rhythmic knee lift exercise often performed in group training situations. Clearly, the characteristics and requirements of the relatively slow-paced trunk tilt exercise performed in the earlier studies and the rhythmic knee lift exercise (e.g., marching in place) with many participants deviate from each other in many respects. For example, the precise postural mimicry of the angular magnitude of the instructor's movement considered essential in the earlier trunk tilt studies was not recognized as a central factor in the rhythmic knee lifting task. This is because comfortable lift height range may be widely varied within the exercise group depending on the participants' physical condition. Accurate temporal mimicry enabling rhythmic synchrony, however, is essential in this type of an exercise in order to keep the group in the same phase. So, in contrast to the instructions given in balance training, it is more vital to communicate the start time rather than end point of the movement. Another change considered necessary for the current investigation was the replacement of the virtual avatar used in [14] by a human instructor to meet the facilities of a typical group exercise situation. Importantly, we also addressed the exercisers' subjective experiences on how they considered touch information in the context of physical exercise.

We designed a system that measured an instructor's leg movements and, on the basis of these measurements, tactile stimulations were wirelessly routed to a leg of a participant to instruct the moments to perform leg lifts. Two versions of a wireless and wearable prototype system were designed in an iterative fashion for investigation. In Study 1, the first prototype version was tested in controlled laboratory conditions with individual adults participating in a simulated exercise situation to get basic information on the functionality of the system and to reveal main development areas. In Study 2, the second prototype version with modified construction and increased functionality needed in actual training situation was tested in realistic conditions with a potential target group consisting of geriatric participants taking part in a structured group exercise. In both studies the participants performed mimicry-based tasks with visual and tactile guidance and then evaluated their experiences of performing the exercise.

\section{Study 1: Laboratory Experiment}

2.1. Introduction. The aim of the first experiment was to evaluate how a wireless tactile guidance application functions in comparison to conventionally used visual demonstration in a knee lift exercise. Typically in such exercise an instructor shows how and when a knee should be lifted, and the exerciser's task is to mimic the demonstration movements in the same phase with the instructor.

We used prerecorded video instructions in giving the visual guidance stimuli to provide consistent and comparable test conditions. A reflection symmetry motor paradigm by Bavelas et al. [16] was used to instruct the participant to imitate the instructor's demonstration movements shown in the video so that the participant and the instructor were facing each other and the task was to respond to the instructor's knee lift as if watching one's own image reflected by a mirror.

Tactile guidance was provided with vibration stimulation on a leg. Tactile stimulus was based on a push metaphor $[11,17]$. This means that the task was to withdraw the leg 
away from the stimulus located in the dorsal side of the leg above crook as though the vibration would "push" the limb to an opposite direction. The stimulus-response compatibility between push versus pull stimulation and consecutive body movements has been studied to some extent (e.g., for wrist rotation direction studied in Jansen et al. [17]), but so far established guidelines for optimal stimulus locations, for example, for a knee lift movement, seem to be nonexistent. Location, duration, vibration frequency, and amplitude of the tactile stimulation were chosen based on a pilot test where six participants evaluated vibratory stimulations in different leg locations while performing a knee lift exercise. The overall preference of the two metaphors was almost equally distributed and thus the decision was based on the slightly more affirmative stimulus amplitude ratings given to the push metaphor stimulations.

There were three modality conditions to provide knee lift instructions: visual $(\mathrm{V})$, tactile $(\mathrm{T})$, and visual-tactile $(\mathrm{VT})$ guidance. Knowing that a slight delay between the onsets of visual and tactile stimuli will inevitably be present due to an acceleration threshold-based triggering of the tactile stimulus (i.e., the instructor's leg movement needed to exceed a certain magnitude to set on the tactile stimulus in the exerciser's leg) as well as operating latency of the vibrotactile actuator, we focused on comparing the knee lift RT between the modality conditions. RT was considered as an important measure because synchronous-enough timing between the onsets of instructor's demonstration movement and the tactile guidance stimulus would be a prerequisite for a successful group exercise especially with visually impaired exercisers. We also investigated possible effects of tactile stimulation on the leg's acceleration magnitude to study the movement dynamics between the different instruction modalities in more detail. In addition to the objective measures of the knee lift RT and acceleration, the participants' subjective experiences were inquired to assess how they rated the tactile instructions in this type of activity.

\subsection{Methods}

2.2.1. Participants. Twelve voluntary participants (3 female and 9 male) between the ages of 19 and 48 years $(\mathrm{M}=26$ and $\mathrm{SD}=9$ ) took part in the study after signing informed consent forms. They were students or staff of the University of Tampere. All reported having normal sense of touch and normal or corrected to normal vision. Eleven of them were right legged and one was left legged by their own report. The study was approved by a local research ethics committee.

2.2.2. Stimuli. The knee lift guidance stimuli were presented in three modality conditions. In $\mathrm{V}$ modality condition the stimuli were provided with an exercise video $(320 \times 240$ pixel resolution, $\sim 30 \mathrm{~Hz}$ sampling rate) where an instructor facing towards the participant produced the stimuli by demonstrating left knee lift movements (Figure 1).

In VT modality condition, a $200 \mathrm{~ms}$ long and $285 \mathrm{~Hz}$ vibration stimulus in the participant's right thigh was coupled with each knee lift event presented in the exercise video.

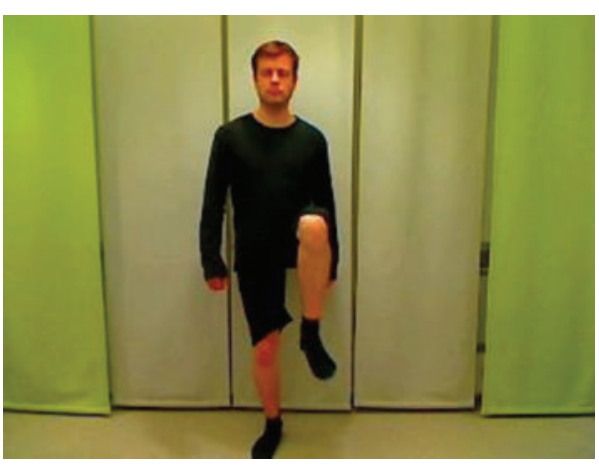

FIGURE 1: A screenshot of the exercise video showing the instructor performing a demonstration movement.

The current stimulus duration has been earlier used in wearable vibration systems for body movement instructions by, for example, Sienko et al. [9] and Jansen et al. [17]. The vibration frequency was within the best sensitivity range of Pacinian corpuscle mechanoreceptors around $10-500 \mathrm{~Hz}$ [18] and was found to be well perceived in the preexperimental pilot test. The vibration was provided in an early phase of the demonstration knee lift movement as soon as the instructor's leg acceleration exceeded a triaxial sum vector threshold of $0.75 \mathrm{~g}$ calculated as

$$
\sqrt{\left(g X^{2}\right)+\left(g Y^{2}\right)+\left(g Z^{2}\right)}
$$

in a baseline-corrected data. The baseline was the mean of the first 200 samples from the beginning of the measurement while the instructor was standing still. The threshold was determined by inspecting accelerations of the demonstration movements shown in the exercise videos. The data showed that the current threshold was required to be exceeded in order to reliably detect the upward phase of each of the demonstration movements. In consequence of the acceleration threshold there was an average of $203 \mathrm{~ms}$ (SD = $31 \mathrm{~ms}$ ) delay between the instructor's initial leg movement and the onset of the tactile stimulus. In T modality condition the vibration stimuli were identical to those in VT, but the visual instructions (i.e., the exercise video) were excluded. In order to reduce the predictability of stimulus onset time, interstimulus interval (ISI) was varied randomly within three durations: 3 (short), 6 (medium), and 9 (long) seconds, approximately. Three separate exercise videos (i.e., one for each modality condition) with differently arranged ISI occurrence were used to eliminate possible learning effect regarding the running order of the short, medium, and long ISI trials.

2.2.3. Apparatus. The first prototype device (Figure 2) was built on Arduino Duemilanove prototyping board that was used for processing the data from an accelerometer, controlling a vibration motor and communicating wirelessly with a PC over Bluetooth. The prototyping board, Bluetooth module, and $9 \mathrm{~V}$ battery used to power the device were enclosed to a box and placed into a belt bag. Precision Microdrives 


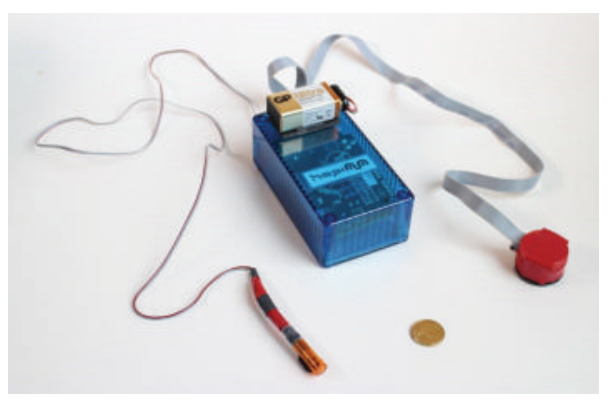

(a)

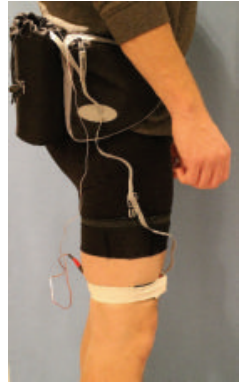

(b)

Figure 2: (a) Wearable parts of the apparatus including electronics in the enclosure box (middle), accelerometer inside the round housing (right), and vibration motor inside the tube housing (left). Scale is indicated by a coin in diameter of $20 \mathrm{~mm}$. (b) The belt bag containing the enclosure box and the leg band equipped with the accelerometer and vibration motor worn by a participant.

vibration motor (model 304-111) encapsulated in a plastic tube was used for stimulating a leg. The typical operating characteristics of the vibration motor included a lag time of $16 \mathrm{~ms}$ and a rise time of $28 \mathrm{~ms}$. A $3.3 \mathrm{~V}$ output of the Arduino board was used together with a bipolar transistor to drive the vibration motor. The transistor was controlled over the digital output of the prototyping board. Dimension Engineering DEACCM3D triaxial accelerometer sensor encapsulated in a plastic housing measured the acceleration of a participant's leg movement. The accelerometer featured a $\pm 3 \mathrm{~g}$ sense range and $500 \mathrm{~Hz}$ bandwidth for $x$-axis, $y$-axis, and $z$-axis. An analog input of the prototyping board was used to read the output of the accelerometer. The vibration motor and the accelerometer were attached to a leg band. The PC and the prototype device communicated with the BlueSMiRF Silver module over serial port. A USB-Bluetooth adapter was used for translating the wireless communication in a sampling rate of $60 \mathrm{~Hz}$. The PC was used for sending the tactile instruction stimulation and presenting the video instructions to the participant and for storing the received acceleration data from the participant's leg using custom software programmed in Processing.org language. The software was used for receiving acceleration data over the wireless communication from the prototype device and for storing the time stamped data to a file for later analysis. The instructions were based on prerecorded acceleration measurements that were recorded with the device and stored together with the recorded exercise video in synchronization. Visual instructions were presented through a $24^{\prime \prime}$ Samsung SyncMaster display.

2.2.4. Procedure. After wearing the belt bag including the electronics, the leg band was fastened around the participant's right thigh so that the accelerometer was located in the ventral side about $10 \mathrm{~cm}$ above the kneecap and the vibration motor was in the dorsal side of the thigh (Figure 2(b)). Then a hearing protector was put on and two $200 \mathrm{~ms}$ long test stimuli were presented to confirm that the participant was able to feel the vibration and unable to hear the vibration sound. The participant was guided to about $120 \mathrm{~cm}$ distance from the computer display of which center point was $155 \mathrm{~cm}$ above the floor. Then the experimenter gave verbal instruction and a visual example of the exercise movement. The task was to lift the right knee until the thigh angle was parallel with the floor, at the maximum, and immediately after that to lay the leg down back in the starting position. The lift was instructed to be done as soon as the visual instruction on the video and/or tactile stimulus was perceived. This instruction was given once before the actual experimental trials. Running order of V, T, and VT conditions and the three exercise videos was fully counterbalanced between the participants. A similar procedure was followed in V, T, and VT conditions apart from the following attributes:

(i) In $\mathrm{V}$ condition, the video was turned on and the vibration motor off.

(ii) In $\mathrm{T}$ condition, the video was turned off and the vibration motor on.

(iii) In VT condition, both the vibration motor and the video were turned on.

After the experimenter had left the room, each condition started with an initiation trial excluded from the analysis and was followed by 21 experimental trials consisting of seven short, medium, and long ISI trials. An experimental trial started with a randomly chosen short, medium, or long ISI which was followed by $\mathrm{V}, \mathrm{T}$, or VT instruction stimulus depending on the modality condition. The participant's task was to immediately respond to the instruction stimulus by a knee lift and then start to wait for a next trial. One condition took about 2.5 minutes. After completing a condition, the experimenter returned to the testing room and the participant was allowed to have a short break. Then the experimenter announced the instruction modality of the upcoming condition. After performing all the three modality conditions the participant assessed them with rating scales. In accordance with Mehrabian and Russell [19] and Bradley and Lang [20] the scales were bipolar nine-point scales varying from -4 to +4 . Ratings were asked for the pleasantness of the task (varying from unpleasant to pleasant), easiness of the task (varying from difficult to easy), and interest of the task (varying from boring to interesting). On each scale, 0 represented a neutral experience (e.g., neither unpleasant nor pleasant). Ratings were given using pen and paper. 
Finally, the participant was asked to rate the tactile stimulation regarding its amplitude (too weak, appropriate, or too strong) and duration (too short, appropriate, or too long). In addition, the temporal uniformity of the timing of the tactile stimulations in relation to the visual instructions (too early, appropriate, or too late) presented in the VT condition was asked to validate the subjective experience of the lag caused by the technical implementation of the tactile stimulus.

2.2.5. Data Analysis. RTs and peak accelerations of the knee lifts were analyzed with a one-way repeated measures analysis of variance (ANOVA) with modality condition as a withinsubjects factor. If the sphericity assumption of the data was violated, Greenhouse-Geisser corrected degrees of freedom were used to validate $F$ statistic. Bonferroni corrected pairwise $t$-tests were used for post hoc tests. RTs were measured from the moment when the instructor's demonstration knee lift exceeded the $0.75 \mathrm{~g}$ acceleration threshold to the moment when the participant's knee lift movement reached its peak acceleration. Peak acceleration sum vector was defined as the highest value during an upward phase of the participant's knee lift event using a similar baseline definition and formula as in determining the threshold for stimulus onset in the exercise video. A total of $33 \%$ of the RT and acceleration data (i.e., 4, 5, and 3 participants in V, T, and VT conditions, resp.) was excluded due to technical reasons causing noisy acceleration signal and replaced by a condition specific series means in the analyses. Prior to the imputation, the normality of the obtained RT and peak acceleration data was confirmed using Shapiro-Wilk test of normality. The tests suggested that the assumption of normal distribution was met in the $\mathrm{V}, \mathrm{T}$, and VT conditions regarding RT $(\mathrm{S}-\mathrm{W}=0.95, \mathrm{df}=8$, and $p=0.67 ; \mathrm{S}-\mathrm{W}=0.95, \mathrm{df}=7$, and $p=0.76 ; \mathrm{S}-\mathrm{W}=0.97, \mathrm{df}=9$, and $p=0.91$, resp.) and peak acceleration data $(S-W=0.90$, $\mathrm{df}=8$, and $p=0.28 ; \mathrm{S}-\mathrm{W}=0.95, \mathrm{df}=7$, and $p=0.72 ; \mathrm{S}-\mathrm{W}=$ 0.87 , $\mathrm{df}=9$, and $p=0.13$, resp.).

Ratings of pleasantness, easiness, and interest were analyzed with three separate Friedman tests. If the Friedman test showed a significant effect, Wilcoxon Signed-Rank tests were used for pairwise comparisons.

2.3. Results. Means and standard error of the means (SEMs) for the RTs and peak accelerations of knee lifts are presented in Figure 3. Nobody missed any knee lift stimuli in the V, T, and VT conditions.

2.3.1. Reaction Times. A one-way repeated measures analysis of variance (ANOVA) showed a statistically significant effect of modality: $F(1.27,14.00)=15.66 ; p \leq 0.001$. Post hoc pairwise comparisons between the modalities showed that the RTs were significantly faster in V and VT conditions as compared to $\mathrm{T}$ condition: $\mathrm{MD}=158 \mathrm{~ms}$ and $p<0.05$ and $\mathrm{MD}=183 \mathrm{~ms}$ and $p<0.001$, respectively. $\mathrm{RT}$ difference between V and VT conditions was statistically nonsignificant.

2.3.2. Peak Accelerations. A one-way ANOVA showed a statistically significant effect of modality: $F(2,22)=5.91 ; p<$ 0.01 . Post hoc pairwise comparisons between the modalities

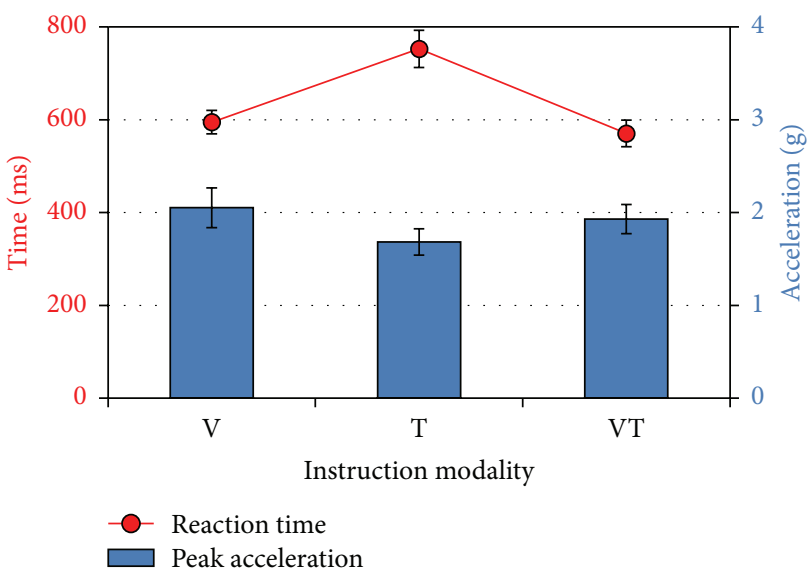

FIGURE 3: Mean RTs and mean peak accelerations of knee lifts in visual (V), tactile (T), and visual-tactile (VT) modality conditions. Error bars represent SEMs.

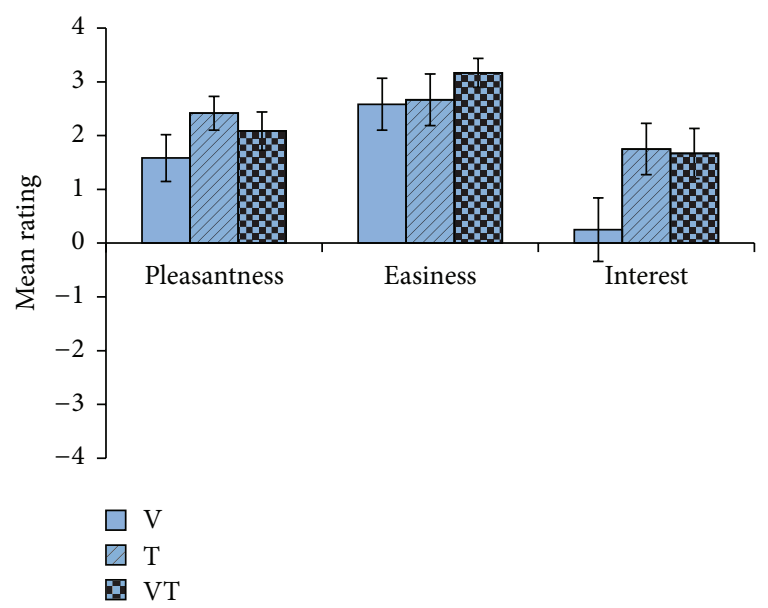

FIGURE 4: Mean ratings for pleasantness, easiness, and interest of the knee lift tasks in visual (V), tactile (T), and visual-tactile (VT) modality conditions. Error bars represent SEMs.

showed that the peak leg accelerations were significantly greater in VT condition when compared to T condition: MD $=0.25 \mathrm{~g}$ and $p \leq 0.05$. Other pairwise comparisons were statistically nonsignificant.

2.3.3. Subjective Ratings. Means and SEMs of the subjective ratings are shown in Figure 4. Friedman test showed a statistically significant effect for the ratings of interest: $\chi^{2}=9.66 ; p<0.01$. For the ratings of pleasantness and easiness, the Friedman test revealed no statistically significant effects. Wilcoxon Signed-Rank tests showed that the task was considered in $\mathrm{T}$ and VT conditions as significantly more interesting than in $\mathrm{V}$ condition: $Z=-2.51$ and $p<0.05$ and $Z=-2.45$ and $p<0.05$, respectively. Difference between $\mathrm{T}$ and VT conditions was not statistically significant.

Vibration amplitude was considered as appropriate by each of the twelve participants. No one reported the vibration being too weak or too strong. Vibration duration was assessed as appropriate by eleven participants, while one considered 
that the vibration was too long. Nobody considered the vibration too short. In VT condition the vibration timing (i.e., synchrony) with respect to the knee lift shown in the instruction video was agreed as appropriate by five participants and the remainder considered that the vibration came too late. Nobody thought that the vibration was too early with respect to the video.

2.4. Discussion. The results of Study 1 showed that tactile instruction channel was functional in a knee lift exercise both alone (i.e., $\mathrm{T}$ condition) and when combined with visual instructions (i.e., VT condition). It seems that the parameters (i.e., duration, vibration frequency, amplitude, and location) of the tactile stimulus were applicable because nobody missed any tactile stimuli. In addition, the findings in the subjective ratings and postexperimental interviews showed favorable assessments of tactile instructions. In the interviews stimulus amplitude and duration were considered as appropriate almost unanimously. The temporal synchrony of the visual and tactile instruction in VT condition, however, polarized the opinions, as more than half of the participants thought that the vibration came too late with respect to knee lift shown in the video.

The experienced asynchrony was likely due to the relatively robust acceleration threshold causing the $203 \mathrm{~ms}$ delay and the $16 \mathrm{~ms}$ onset latency of the vibration actuator, which together resulted in a minimum of about $220 \mathrm{~ms}$ delay from the instructor's initial leg movement to the onset of the tactile stimulus in the participant's leg. In consequence, the instructor's leg movement was already visible in the video when the tactile stimulus started. The delay can also explain why the mean RT (i.e., time to reach the peak leg acceleration) in $\mathrm{T}$ condition was 158.2 and $182.5 \mathrm{~ms}$ slower as compared to $\mathrm{V}$ and VT conditions, respectively.

If the tactile stimulus onset had been created in exact synchrony with the instructor's initial leg movement, the RTs in $\mathrm{T}$ and VT conditions could, in fact, have been about 40$60 \mathrm{~ms}$ faster than those in $\mathrm{V}$ condition. This conclusion is based on subtraction of the lag caused by the technical implementation from the mean RT of T condition and supported by earlier findings showing about $30-40 \mathrm{~ms}$ shorter RTs to tactile than visual stimuli (e.g., $[3,5,21]$ ). Following this, tactile instructions would seem to have the potential to enable even faster knee lift reactions than conventionally used visual instruction provided that the onset of the tactile stimulus could be reliably situated to the initial phase of the instructor's leg movement. However, the aim of the present study was to assess the functionality of tactile instruction system in a realistic exercise situation, where a robust-enough onset method would be required to prevent the instructor from giving false-positive instruction stimuli resulting, for example, from minor or unintentional leg movements between the repetitions. Thus, lowering the acceleration threshold to diminish the onset delay would require further studies to find an acceptable trade-off between the speed and accuracy of providing the tactile instructions.

Instruction modality also had an effect to the acceleration magnitude of the leg movements. VT instruction resulted in significantly higher peak acceleration than $\mathrm{T}$ instruction. Despite the fact that the mean peak acceleration was the highest in $\mathrm{V}$ condition and the lowest in $\mathrm{T}$ condition, the difference did not reach statistical significance. As it can be seen in the SEM error bars of Figure 3, this was due to the larger deviation of acceleration magnitude in $\mathrm{V}$ condition compared to those found in $\mathrm{T}$ and $\mathrm{VT}$ conditions. This result may indicate that tactile stimulation stabilized the acceleration of leg movement and enabled more consistent repetitions.

Ratings of the pleasantness, easiness, and interest of the exercise task were clearly positive in each modality condition which indicates that tactile instruction channel was well accepted in the current context along with conventionally used visual instructions both unimodally and when combined with visual instruction modality. This was despite the fact that the participants were fully capable of following visual instructions and, therefore, did not necessarily need tactile instructions in this type of activity. Moreover, tactile stimulation provided in $\mathrm{T}$ and $\mathrm{VT}$ conditions increased significantly the participants' interest of the task when compared to $\mathrm{V}$ condition. This finding suggests that such a device may be of interest to exercisers also in real training situations, which in turn would be important in the light of earlier studies showing that interest towards the training is a central factor in increasing the motivation and exercise adherence (e.g., [22]).

\section{Study 2: User Experience and Acceptance of a Vibrotactile Exercise Device among Elderly Participants}

3.1. Introduction. In a follow-up user study, the wearable tactile device used in Study 1 was iterated further and redesigned to be used in a group exercise situation with elderly participants. This approach was chosen based on a recent trend that has been shifting increasingly from oneto-one personal training to group exercise and training programs [23]. Supervised group exercise is often favored in public and institutional healthcare, because it is clearly more time-effective and cost-effective in comparison to personal training and it has been found to be beneficial in promoting not only physical fitness [24] but also social and mental wellbeing and overall quality of life [25].

A new version of the device allowed an instructor to activate an exerciser's legs with tactile stimulation automatically and in real time (i.e., in conjunction with the instructor's demonstration movements) via a wireless link. The main goal of this user study was to evaluate acceptance and user experience (UX) of the tactile exercise device in a context of recreational group exercise among a potential target population. The approach had three main research questions:

(1) How do elderly participants experience the use of a tactile device in a group exercise context?

(2) How does it differ from conventional session without tactile instructions? 


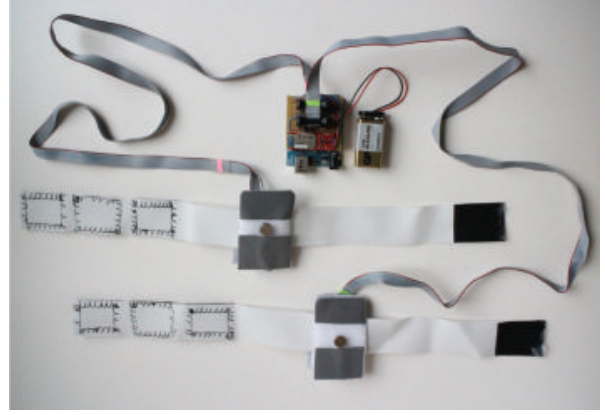

(a)

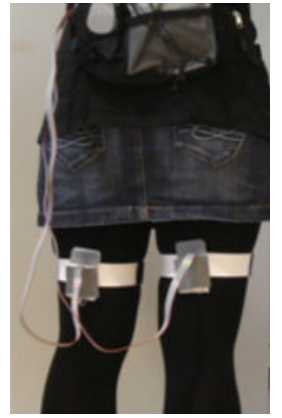

(b)

Figure 5: (a) Wearable parts of the exercise device and (b) their attachment locations.

(3) Do the elderly participants accept the use of tactile stimulation in a group exercise context?

The user study consisted of exercise sessions both with and without the device, UX questionnaires, and interviews inquiring the exercisers' as well as the instructors' impressions, experience, and acceptance of using the device.

\subsection{Methods}

3.2.1. Participants. Participants and premises for the study were provided by a service center for elderly people. The targeted user group consisted of relatively healthy elderly volunteers without any major cognitive disabilities to ensure that they were fully able to answer questionnaires and take part in an interview. They were also required to be able to take part in the physical exercise tasks, including knee lifting used in Study 1. Two male and six female exercisers and two female instructors took part in the study after signing informed consent forms. The exercisers were between the ages of 73 and 92 years $(M=83$ and $S D=7)$. The instructors were 27 and 39 years old. One of the instructors had a formal education in physical therapy. The study consisted of two within-subjects exercise groups, each with one instructor and four exercisers. The study was approved by the local research ethics committee.

3.2.2. Apparatus. The tactile device consisted of two elastic leg bands and a belt bag (Figure 5). Both the leg bands included a tactile actuator and accelerometer, while the belt bag contained an Arduino board and a $9 \mathrm{~V}$ battery. The components in the leg bands were connected to the board via flat cables. The study was done with two identical devices. One device worn by an instructor functioned in a "master" mode and the other worn by an exerciser was set in a "slave" mode. The devices communicated via Bluetooth with a PC acting as host and routing the communication between the devices. A more detailed description of the system's operation principle is given in [26]. The major change from the users' view point was the fact that, contrary to the device used in Study 1 , leg bands were attached to both limbs. In addition, the vibration actuator type was replaced with Solarbotics VPM2 Vibrating Disk Motor due to its closed structure.
There was a mechanical delay of $60 \mathrm{~ms}$, on average, measured from the onset of the applied voltage $(3 \mathrm{~V})$ to the beginning of the actuation from the motor using a GW Instek GDS2104A oscilloscope. The accelerometers were relocated above the actuators in the dorsal side of the legs. Together these revisions enabled a more compact design of the leg bands.

3.2.3. User Experience Questionnaire. The UX questionnaire was used for studying the research questions 1 and 2 . The questionnaire had two variants: one for evaluating the exercise experience while wearing the device and another without it. They had nearly identical scales and distinctive instructions on what to evaluate. The questionnaires were based on UX model by Hassenzahl [27]. The semantic differentials were selected from two different questionnaires: AttrakDiff 2 [28] and Attrak-Work [29]. AttrakDiff 2 questionnaire consists of seven-point semantic differential scales, which are divided into pragmatic qualities (PQ), hedonic qualities of stimulation (HQS) and identification (HQI), and attractiveness (ATT). The Attrak-Work adds a task and goal achievement (TGW) quality, which is essential in work-related contexts. The pragmatic scales are close to the traditional definition of usability (usefulness, utility, and usability). If a product is stimulating, it provides possibilities to gain new knowledge and self-development, while identification can be explained as social self-expression through physical objects and possessions. Three device-centric scales were omitted from the questionnaire without the device: impractical, practical; makes exercise harder, makes exercise easier; and slows down the exercise, speeds up the exercise. A few scales were selected for each of the five themes to keep the questionnaire length on one page. Moreover, some scales had to be left out as they were presumed to be inapplicable to this study due to the context and nature of the device. The selected scales for each quality are presented in Table 1.

3.2.4. Interview. The group interviews concentrated on qualitative information on the participants' general impressions of tactile instructions and the device. The interviews were semistructured and started with free-form description of the participants' impressions of the user experience. This was followed by a discussion about the exercise with and without 
TABLE 1: Qualities and scales used in the UX questionnaires.

\begin{tabular}{|c|c|c|}
\hline Quality & Scale & ID \\
\hline \multirow{4}{*}{ Pragmatic } & Complex, simple & PQ_2 \\
\hline & Impractical, practical & PQ_3 \\
\hline & Challenging, effortless & PQ_4 \\
\hline & Confusing, clear & PQ_6 \\
\hline \multirow{3}{*}{$\begin{array}{l}\text { Hedonic: } \\
\text { stimulation }\end{array}$} & Conventional, original & HQS_1 \\
\hline & Unimaginative, creative & HQS_2 \\
\hline & Dull, absorbing & HQS_5 \\
\hline \multirow{4}{*}{$\begin{array}{l}\text { Hedonic: } \\
\text { identification }\end{array}$} & Unprofessional, professional & HQI_2 \\
\hline & Poor quality, high quality & HQI_4 \\
\hline & Noninclusive-Inclusive & HQI_5 \\
\hline & $\begin{array}{l}\text { Separates me from people, brings me } \\
\text { closer to people }\end{array}$ & HQI_6 \\
\hline \multirow{4}{*}{ Attractiveness } & Unpleasant, pleasant & ATT_1 \\
\hline & Unattractive, attractive & ATT_3 \\
\hline & Bad, good & ATT_5 \\
\hline & Discouraging, motivating & ATT_7 \\
\hline \multirow{3}{*}{$\begin{array}{l}\text { Task and goal } \\
\text { achievement }\end{array}$} & $\begin{array}{c}\text { Makes exercise harder, makes exercise } \\
\text { easier }\end{array}$ & TGW_1 \\
\hline & $\begin{array}{c}\text { Slows down the exercise, speeds up the } \\
\text { exercise }\end{array}$ & TGW_4 \\
\hline & Difficult to control, easy to control & TGW_7 \\
\hline
\end{tabular}

the device, acceptance, and other ideas for the device and applications both the exercisers and instructors might have.

3.2.5. Procedure. The exercisers' task was to follow and repeat the exercise guided by the instructor. The exercise consisted of moving legs in seated and standing positions, including various leg lifting tasks, stationary marching, and dancing. The whole exercise was done with background music. The sessions were designed and carried out by the instructors as normally as possible but with emphasizing on legs and lower part of the body. The exercise sessions began with an introduction to the purpose of the user study and a brief explanation of the device. The exercisers were told that the instructor's device sent tactile instructions to the exerciser's device as an additional signal to start performing the exercise movements. Tactile stimuli were produced concurrently in both the instructor's device and the exerciser's device, thus enabling the instructor to confirm a successful sending of a tactile instruction. Due to the limited amount of devices, the exerciser's device was rotated between the four exercisers in the group. Every 15 minutes the exercise was paused to switch the device to a next exerciser. As a result, each exerciser got to wear and exercise with the device for about 15 minutes during the 1-hour session. The instructor wore the other device for the whole exercise session. Halfway through the session, the exercisers filled the UX questionnaires evaluating the subjective experience of exercising in the group. The first two exercisers to try the tactile device filled the questionnaire "exercise with the device," and the other two filled the questionnaire "exercise without the device." This was repeated at the end of the session but this time vice versa. At the end, each exerciser in the group evaluated the exercise with and without wearing the device. The instructors in both groups evaluated the user experience of exercising with the device after the session. Following the session, the group (including the instructor) took part in 30-minute-long interview.

\subsection{Results}

3.3.1. User Experience Questionnaire. The exercisers' ratings given to the UX questionnaire (Figure 6(a)) showed that the exercise both without and with the device was experienced mainly in a positive way. They evaluated the exercise with the device to be more motivating than that without the device (ATT_7), but otherwise the SEM ranges of the ratings were overlapping between the two exercise situations indicating potentially nonsignificant differences. Ratings of the three items reflecting the device use situation only were also positive; the exercisers considered that the device was more practical than impractical (PQ_3), made the exercise somewhat easier (TGW_1), and speeded up the exercise (TGW_4). Interestingly, the instructors' ratings of the device in Figure 6(b) were mainly outside the SEM range of those given by the exercisers shown in Figure 6(a). One or both of the instructors rated the device more positively than the exercisers regarding 8 items (i.e., PQ_2, PQ_6, HQS_1, HQS_2, HQI_2, HQI_5, HQI_6, and ATT_7) and more negatively regarding 10 items (i.e., PQ_3, PQ_4, HQI_4, ATT_1, ATT_3, ATT_5, ATT_7, TGW_1, TGW_4, and TGW_7). Consensus was found only for item HQS_5; the exercise with the device was found equally absorbing by the exercisers and the two instructors. However, no statistical analysis was performed for the questionnaire data due to low number of the participants.

3.3.2. Interview: Exercise Experience with and without the Device. Exercisers' expectations of the exercise with the device were summarized into three groups: (1) no expectations, (2) slightly nervous or afraid, and (3) interested. Majority of the exercisers were slightly nervous or afraid of trying a device they did not have any experience with. However, after the exercisers tried the device, many of the expectations were changed in a positive way as exemplified by following quotations:

\section{"I was nervous about this thing we were supposed to wear. Then I saw how simple it was and the fear vanished," an exerciser said. \\ "At first I was nervous and surprised. I didn't expect it to be this easy," an exerciser said.}

On the other hand, one exerciser commented that his/her expectations did not change, and another one had expected much stronger stimulation.

First impressions of exercise with the tactile device, for both the exercisers and instructors, were positive and even surprising. These consisted of positive and neutral descriptions, such as nice, pleasant, easy, fun, ordinary, different (i.e., 


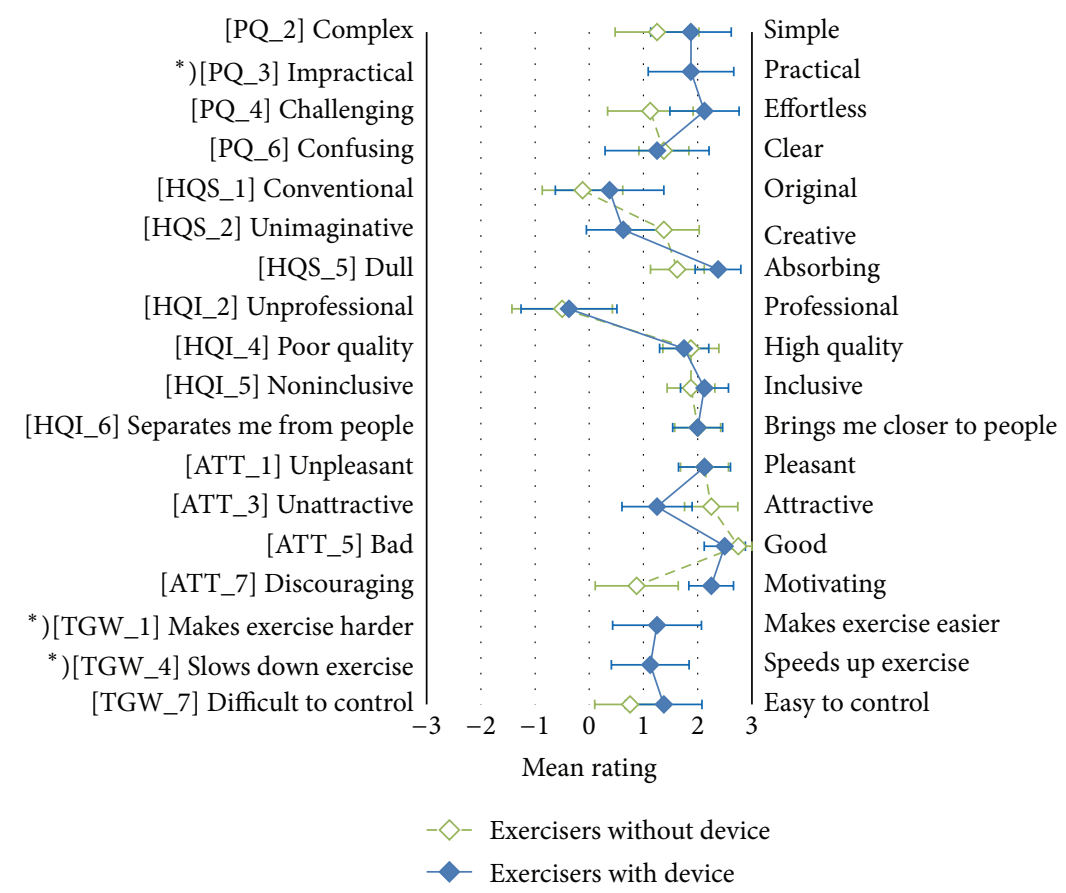

(a)

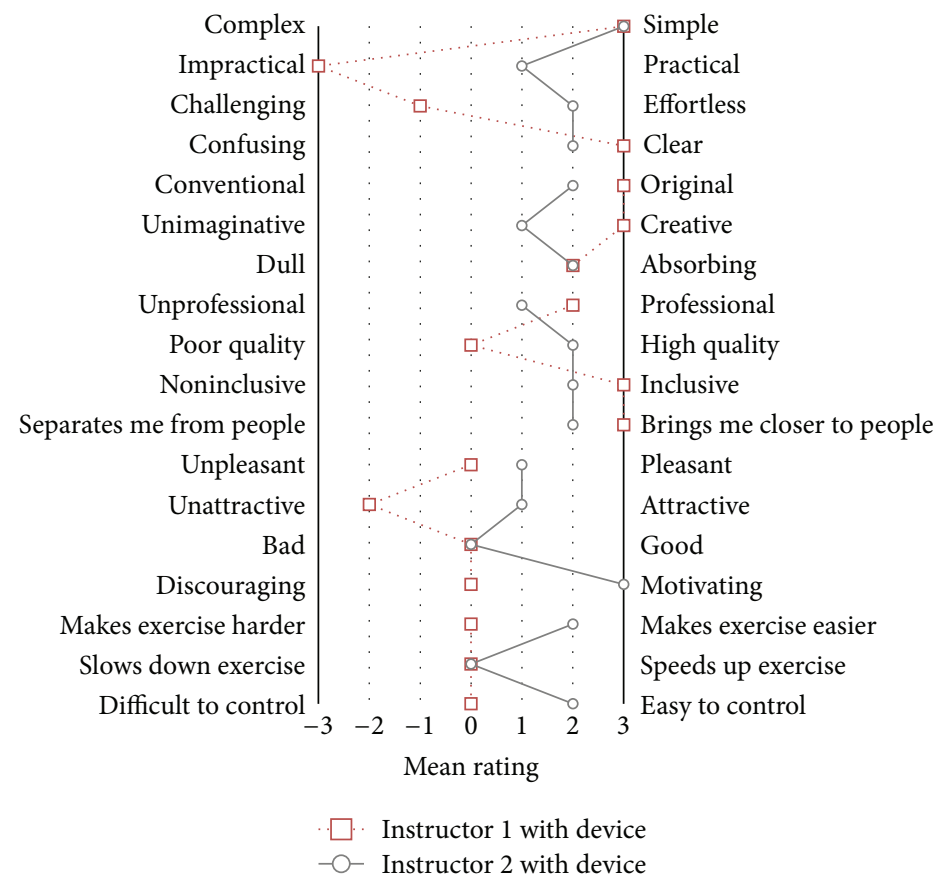

(b)

FIGURE 6: Mean ratings for the UX questionnaires on 7-point semantic differential scale given by (a) the exercisers and (b) the instructors. Items labeled with *) in (a) were excluded from the "exercise without the device" questionnaire. Error bars in (a) represent SEMs.

something new), and effortless. The emphasis on the easiness was frequent in the comments. Once the device was put on, the exercisers did not need to concentrate on operating the device. The majority of the exercisers also quickly understood the purpose of the device with a very short introduction. Two of the exercisers needed further instructions. One exerciser had major difficulties in recognizing the tactile stimulation and another was so concentrating on following the tactile instructions that she forgot to pay attention to the actual exercise. The tactile stimuli and the form-factor of the wearable device were comfortable and pleasant according to the exercisers. However, one of the instructors said that wearing the device for an hour became uncomfortable because of the frequent tactile stimulation. 
In addition to the positive reaction, the tactile exercise device seemed to bring something new to the exercise. Variation to the usual exercise was considered positive and refreshing. The exercisers and instructors said that the device encourages and motivates one to exercise. The instructors explained that the device also created a new kind of bond between them by enabling remote and hands-free touch stimulation. Even though the exercisers reported mainly focusing on the visual and auditory guidance, the tangible and intuitive feel of the rhythm of the exercise was said to be motivating. Citations below illustrate new possibilities and insights inspired by the device:

\section{"I teased a little bit by sending [tactile] stimulation also outside the exercise tasks," an instructor said.}

\section{"I instinctively moved my leg when I got the [tactile] stimulation," an exerciser said.}

3.3.3. Interview: Wearing and Using the Device. Although the exercise experience was mainly positive, the actual device got mixed feedback. The device was found usable in both seated and standing positions, which is important for the groups consisting of elderly participants with varying physical conditions. Although the leg bands and waist-mounted electronics were connected with cables, it did not restrict the movements during the exercise. As a drawback, the exercisers needed help in putting the device on.

The instructors stated that the device was capable of displaying slow rhythm to the exercisers, but faster rhythms were not distinguishable as the device would then vibrate constantly. The delay between the two devices was also said to be too long and creating an out-of-sync issue between the different instruction modalities.

The main problem recognized was the stimulation model of the current implementation, which provided tactile stimulation only for a limited set of leg exercise movements. Slow and static exercise moves (e.g., when the leg is moved very slowly and kept still to exercise muscles) resulted in either excess stimulation or no stimulation at all. In consequence of the simple and robust stimulation model, the usefulness of the device was found limited in the exercise context of this study.

3.3.4. Interview: Acceptance. Despite the encouraging user experience, the interview questions related to acceptance did not get as positive responses. The responses were divided by the groups: the first group would not use the tactile device in exercise and background music was preferred over it and the second group would use the device occasionally to bring variety to the exercise groups. Both groups were suspicious of the actual benefits of using such system. The delay and constricted stimulation model issues were also raised as a major drawback affecting the acceptance. However, the exercisers and instructors also commented that the device should be used more comprehensively to be able to better evaluate its acceptance and usefulness:

\begin{abstract}
"If used too often, it might create a feeling that I cannot do the exercise without aiding devices," an exerciser said.
\end{abstract}

"Would bring variety and sustain the interest in
the exercise if the device is used from time to time,"
an exerciser said.

3.3.5. Interview: Further Development and Research Ideas. The interviews spawned few development ideas for the device. Overall, the device should support a more comprehensive set of exercise moves. The instructors proposed a stimulation model based on the activation of large muscle groups for slower paced muscle exercises. Also the information about range of motion would be useful, as one of the exercise goals is to improve it on the elderly exercisers. It was also suggested that the delay should be minimized or the tactile stimulation could be generated based on the rhythm of the music to avoid delay issues. Finally, amplitude of the tactile stimulation could be adjustable to suit the exercisers' varying levels of touch sensitivity. The potential of the device was identified also in remote exercise context where some or all exercisers can be in different locations.

3.4. Discussion. The results of Study 2 showed that the device got mostly positive responses from the elderly exercisers. However, the current implementation of the stimulation method (i.e., onset of tactile stimulus was triggered based on the instructor's leg acceleration data) caused slight delay and thus did not support real-time exercise situation very well. In particular the instructors brought up the delay as an issue that should be further developed.

The user study resulted in encouraging user experience findings. Regarding the research questions 1 and 2, the device was perceived to be easy to learn and pleasant to use. The UX questionnaire revealed that the exercisers thought that the device increased motivation to exercise. Otherwise the experience of the exercise session was rated quite similarly both with and without the device.

The third research question about acceptance got mixed responses. Usefulness of the device was questioned by the exercisers and instructors. This was probably because all the elderly participants of the current study were in relatively good physical condition and able to follow visual demonstrations and verbal instructions. In consequence, they had no absolute need for tactile instructions. The exercisers preferred to train with background music and were ready to use the device from time to time. For the instructors, the stimulation model, which was restricted to recognize only rapid leg movements, and delay limited the contents of the exercise in excess and thus the device with the current design was not considered very useful within the current context and participants. Based on these findings, future work should concentrate on refining the tactile stimulation model so that it would support better a fast-tempo exercise by minimizing the delay and the fact that the system would function also in slow and long term movements such as muscle stretching. 


\section{General Discussion}

The results of Studies 1 and 2 showed that tactile stimulation can be used successfully in mediating real-time instructions to perform leg movements in simulated laboratory conditions as well as in realistic group exercise situation. Tactile instructions were found applicable in both complementing (Studies 1 and 2) and substituting (Study 1) the typically used visual exercise instructions. With this regard the results are in line with earlier studies showing that tactile stimulation (e.g., vibration) can be practical in various contexts of physical exercise (e.g., [10-12]) and rehabilitation (e.g., [8, 9]). However, typical set up in the earlier concepts has been that tactile stimulation provides feedback derived from the exerciser's own actions in the context of self-contained exercise. In contrast to this, our aim was to study tactile support specifically in a motor-mimicry frame of reference. That is to say, in the current approach, the source and the communicative function of the tactile stimulation was movement of another human being. To our knowledge, along with the work by Lee and colleagues $[14,15]$, this was among the first approaches to study technology-mediated touch information in the context of mimicry-based behavioral regulation in physical exercise. In this framework, our results showed a potential for utilizing real-time tactile communication between a human instructor (in contrast to an avatar used in [14]) and exerciser(s) in rhythmic physical exercise. As an additional complement to earlier work, the current approach obtained the exercisers' subjective experiences.

Physical measurements conducted in Study 1 showed that tactile instruction modality diminished the deviation of peak acceleration magnitude of the knee lift movements in comparison to visual-only instructions. It is noted that this effect took place with healthy and relatively young participants. The result may indicate that tactile stimulation stabilized motor responses and thus enabled better control to perform exercise movements in a consistent way even for exercisers with fully functional sensory system and motor coordination. This could be a useful supplement in various motion training exercises where repeatable and precise control of movement is of the essence. Noisy acceleration data caused rejection of objective data from 3 to 5 participants in the three stimulus conditions. However, each participant performed 21 trials in each condition and thus the total amount of data in one condition was based on relatively high number of 147-189 trials. The stability of the results was indicated by normally distributed values across the RT and acceleration of the obtained data.

Subjective evaluations of Study 1 revealed that tactile instruction modality both alone and when combined with visual instructions was found to be as equally pleasant and easy as visual-only instructions. Importantly, tactile stimulation increased significantly the interest of the exercise task. The delay between the visual instruction and the onset of tactile stimulation reported in both studies showed that the tactile stimulation model was found suitable mainly for simple and slow-paced exercise in its current form. The exercise phase of Study 1 can be regarded as relatively slow as the interval between successive knee lifts varied between 3 and 9 seconds. In these circumstances the temporal synchrony between visual demonstration and concurrent tactile stimulation was experienced as appropriate by nearly half of the participants. Even so, the fact that the slim majority found the delay being too long shows that there is still room for improving the onset model of the tactile stimulus. This finding recurred also in the actual exercise situation of Study 2 , where in particular the instructors commented that the delay limited the pace of the exercise.

In Study 2 the elderly exercisers' general experience of the device was clearly positive and in some respects it improved the experience compared to their normal exercise situation. This finding is in line with earlier results by Mitzner et al. [30] showing that, contrary to popular stereotypes, older adults have mainly positive attitude towards technological devices especially when the technology is easy to use and it supports their activities, enhances convenience, and contains useful features. Applying new interactive technologies (e.g., body controlled gaming interfaces such as Microsoft Kinect) for regular use presumes additionally that the technologies connect with routines and spaces that people inhabit [31]. These conditions were met in the present study seeing that the user interface was virtually invisible for the exercisers and the system was integrated with already familiar activity, space, and people.

Few doubts related to usefulness amongst the participants in Study 2 boiled down to a need for development of the stimulation model and brought up topics for future research. For example, evaluating the usefulness of the device with disabled people (e.g., visually, aurally, and cognitively impaired and those with difficulties in initiating limb movements) would provide more insight into which special groups would potentially benefit most from tactile instruction channel.

In fact, one interesting topic would be a deeper investigation of blind people's imitation behavior using tactile instruction modality. There is evidence that the human mirror neuron system, which is argued to play a central role in visuomotor imitation learning [32], allocates same resources regardless of sensory modality [33]. Findings by Ricciardi et al. [33] demonstrate that visual experience is not a prerequisite for the mirror system development and that even congenitally blind people without vision-based imagery can "see" the actions of others by recruiting the same network of cortical areas activated by action observation in sighted subjects. The authors suggest that the mirror system stores a motor representation of others' actions that can be evoked through supramodal sensory mechanisms. Research on mimicry-based learning activated by tactile stimuli (in comparison to those perceived via visual modality) could open new insights into motor learning amongst the visually impaired.

In addition to tactile instructions, a wide range of physical exercise and therapy methods would benefit from objective measurement and analysis of physical movement. It is known that objective feedback from one's own performance can contribute favorably to learning of motor skills [23] and maintain motivation to continue the training program [34]. However, conventional physical activity monitoring devices, such as pedometers and motion sensors, are incapable of 
assessing exerciser's performance such as RT, intensity, and rhythmic synchrony of body movements with respect to instructor's demonstration movements. In this context, our concept could provide more insight by quantifying exercise performance for both online monitoring and further analysis. This would enable the instructor to observe multiple exercisers' individual performances in an equal manner and to reliably follow their long term progress.

Altogether, there is a clear need for new actions to improve the current situation where ageing people and people having disabilities are at risk of serious health problems associated with lack of physical activity. In USA, for instance, adults with disability are twice as likely to be physically inactive as compared to those with no disability [35], and only about $16 \%$ of the $\geq 65$ years old meet the recommended amount of physical activity $[36,37]$.

Taken together, the current objective and subjective data suggest that a rather simple and inexpensive tactile instruction system could open up possibilities to increase physical activity for those being at risk to be excluded from organized group exercise that typically demands the ability to follow visual and auditory instructions. The possibility to use the device remotely (e.g., from one's home) could help in overcoming transportation difficulties and finally quantified and unbiased information of the performance could give an additional boost and motivate people to continue with regular training.

\section{Conflict of Interests}

The authors declare that there is no conflict of interests regarding the publication of this paper.

\section{Acknowledgments}

Thanks are due to staff and residents of the Kaukaharju service center for their assistance in Study 2. Thanks are also due to Ahmed Farooq for technical assistance in measuring the actuator delay time. This research was part of HAPIMM project funded by the Finnish Funding Agency for Technology and Innovation (Tekes), Decision no. 40159/09, and Doctoral Programme in User-Centered Information Technology (UCIT).

\section{References}

[1] Y. Visell, "Tactile sensory substitution: models for enaction in HCI," Interacting with Computers, vol. 21, no. 1-2, pp. 38-53, 2009.

[2] M. A. Heller, "Development and intermodal relations," in The Psychology of Touch, M. A. Heller and W. Schiff, Eds., pp. 115117, Lawrence Erlbaum Associates, Hillsdale, NJ, USA, 1991.

[3] J. M. T. Brebner and A. T. Welford, "Introduction: an historical background sketch," in Reaction Times, A. T. Welford, Ed., pp. 1-23, Academic Press, New York, NY, USA, 1980.

[4] R. Mohebbi, R. Gray, and H. Z. Tan, "Driver reaction time to tactile and auditory rear-end collision warnings while talking on a cell phone," Human Factors, vol. 51, no. 1, pp. 102-110, 2009.
[5] J. V. M. Hanson, D. Whitaker, and J. Heron, "Preferential processing of tactile events under conditions of divided attention," NeuroReport, vol. 20, no. 15, pp. 1392-1396, 2009.

[6] Á. A. Jakobovits, "Grasping activity in utero: a significant indicator of fetal behavior (the role of the grasping reflex in fetal ethology)," Journal of Perinatal Medicine, vol. 37, no. 5, pp. 571$572,2009$.

[7] G. Dolce and L. Sazbon, The Post-Traumatic Vegetative State, Thieme, Stuttgart, Germany, 2002.

[8] P. B. Shull, K. L. Lurie, M. R. Cutkosky, and T. F. Besier, "Training multi-parameter gaits to reduce the knee adduction moment with data-driven models and haptic feedback," Journal of Biomechanics, vol. 44, no. 8, pp. 1605-1609, 2011.

[9] K. H. Sienko, M. D. Balkwill, L. I. E. Oddsson, and C. Wall, "The effect of vibrotactile feedback on postural sway during locomotor activities," Journal of NeuroEngineering and Rehabilitation, vol. 10, no. 93, 6 pages, 2013.

[10] J. B. Van Erp, I. Saturday, and C. Jansen, "Application of tactile displays in sports: where to, how and when to move," in Proceedings of the EuroHaptics International Conference (EuroHaptics '06), pp. 90-95, Springer, Paris, France, 2006.

[11] D. Spelmezan, M. Jacobs, A. Hilgers, and J. Borchers, “Tactile motion instructions for physical activities," in Proceedings of the 27th International Conference Extended Abstracts on Human Factors in Computing Systems (CHI '09), pp. 2243-2252, Boston, Mass, USA, April 2009.

[12] M. Bächlin, K. Förster, and G. Tröster, "SwimMaster: a wearable assistant for swimmer," in Proceedings of the 11th ACM International Conference on Ubiquitous Computing (UbiComp '09), pp. 215-224, ACM, Orlando, Fla, USA, October 2009.

[13] J. Lylykangas, V. Surakka, J. Rantala, and R. Raisamo, "Intuitiveness of vibrotactile speed regulation cues," ACM Transactions on Applied Perception, vol. 10, no. 4, article 24, 2013.

[14] B.-C. Lee and K. H. Sienko, "Improving balance training via multimodal biofeedback," in Proceedings of the International Conference on Fall Prevention and Protection (ICFPP '10), pp. 77-80, May 2010.

[15] B.-C. Lee, S. Chen, and K. H. Sienko, "A wearable device for real-time motion error detection and vibrotactile instructional cuing," IEEE Transactions on Neural Systems and Rehabilitation Engineering, vol. 19, no. 4, pp. 374-381, 2011.

[16] J. B. Bavelas, A. Black, N. Chovil, C. R. Lemery, and J. Mullett, "Form and function in motor mimicry topographic evidence that the primary function is communicative," Human Communication Research, vol. 14, no. 3, pp. 275-299, 1988.

[17] C. Jansen, A. Oving, and H.-J. van Veen, "Vibrotactile movement initiation," in Proceedings of the EuroHaptics International Conference (EuroHaptics '04), pp. 110-117, Munich, Germany, June 2004.

[18] E. B. Goldstein, Sensation \& Perception, Brooks/Cole Publishing Company, Pacific Grove, Calif, USA, 1999.

[19] A. Mehrabian and J. A. Russell, An Approach to Environmental Psychology, MIT Press, Cambridge, Mass, USA, 1974.

[20] M. M. Bradley and P. J. Lang, "Measuring emotion: the selfassessment manikin and the semantic differential," Journal of Behavior Therapy and Experimental Psychiatry, vol. 25, no. 1, pp. 49-59, 1994.

[21] C. Ho, C. Spence, and H. Z. Tan, "Warning signals go multisensory," in Proceedings of the 11th International Conference on Human-Computer Interaction (HCI International '05), pp. 1-10, Las Vegas, Nev, USA, July 2005. 
[22] R. M. Ryan, C. M. Frederick, D. Lepes, N. Rubio, and K. M. Sheldon, "Intrinsic motivation and exercise adherence," International Journal of Sport Psychology, vol. 28, no. 4, pp. 335354, 1997.

[23] R. B. Shepherd, "Exercise and training to optimize functional motor performance in stroke: driving neural reorganization?" Neural Plasticity, vol. 8, no. 1-2, pp. 121-129, 2001.

[24] E. E. Baum, D. Jarjoura, A. E. Polen, D. Faur, and G. Rutecki, "Effectiveness of a group exercise program in a long-term care facility: a randomized pilot trial," Journal of the American Medical Directors Association, vol. 4, no. 2, pp. 74-80, 2003.

[25] J. J. Eng, K. S. Chu, C. M. Kim, A. S. Dawson, A. Carswell, and K. E. Hepburn, "A community-based group exercise program for persons with chronic stroke," Medicine and Science in Sports and Exercise, vol. 35, no. 8, pp. 1271-1278, 2003.

[26] K. Myllymaa, R. Raisamo, J. Lylykangas, J. Heikkinen, and V. Surakka, "RehApp-a wearable haptic system for rehabilitation and sports training," in Proceedings of the International Conference on Haptics: Perception, Devices, Mobility, and Communication-Volume Part II (EuroHaptics '12), pp. 210-213, Tampere, Finland, June 2012.

[27] M. Hassenzahl, "The thing and I: understanding the relationship between user and product," in Funology: From Usability to Enjoyment, Kluwer Academic Publishers, 2007.

[28] M. Hassenzahl, M. Burmester, and F. Koller, "AttrakDiff: a questionnaire for the measurement of perceived hedonic and pragmatic quality," in Mensch \& Computer 2003: Interaktion in Bewegung, J. Ziegler and G. Szwillus, Eds., B.G. Teubner, Stuttgart, Germany, 2003.

[29] H. Väätäjä, T. Koponen, and V. Roto, "Developing practical tools for user experience evaluation: a case from mobile news journalism," in Proceedings of the European Conference on Cognitive Ergonomics: Designing beyond the ProductUnderstanding Activity and User Experience in Ubiquitous Environments (ECCE '09), Article 23, p. 8, Helsinki, Finland, September 2009.

[30] T. L. Mitzner, J. B. Boron, C. B. Fausset et al., "Older adults talk technology: technology usage and attitudes," Computers in Human Behavior, vol. 26, no. 6, pp. 1710-1721, 2010.

[31] B. Nansen, F. Vetere, T. Robertson, J. Downs, M. Brereton, and J. Durick, "Reciprocal habituation: a study of older people and the Kinect," ACM Transactions on Computer-Human Interaction, vol. 21, no. 3, article 18, 20 pages, 2014.

[32] G. Rizzolatti and L. Craighero, "The mirror-neuron system," Annual Review of Neuroscience, vol. 27, pp. 169-192, 2004.

[33] E. Ricciardi, D. Bonino, L. Sani et al., "Do we really need vision? How blind people 'see' the actions of others," The Journal of Neuroscience, vol. 29, no. 31, pp. 9719-9724, 2009.

[34] A. W. Vaes, A. Cheung, M. Atakhorrami et al., "Effect of 'activity monitor-based" counseling on physical activity and healthrelated outcomes in patients with chronic diseases: a systematic review and meta-analysis," Annals of Medicine, vol. 45, no. 5-6, pp. 397-412, 2013.

[35] J. H. Rimmer and A. C. Marques, "Physical activity for people with disabilities," The Lancet, vol. 380, no. 9838, pp. 193-195, 2012.

[36] Centers for Disease Control and Prevention (CDC), "Suicide among adults aged 35-64 years-United States, 1999-2010," Morbidity and Mortality Weekly Report, vol. 62, no. 17, pp. 326330, 2013, http://www.cdc.gov/mmwr/pdf/wk/mm6217.pdf.

[37] US Department of Health and Human Services, 2008 Physical Activity Guidelines for Americans, US Department of Health and Human Services, Hyattsville, Md, USA, 2008, http:// www.health.gov/paguidelines. 

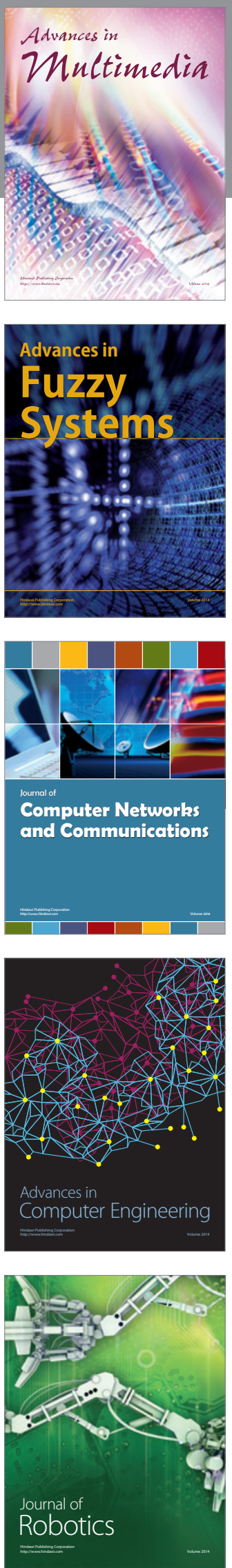

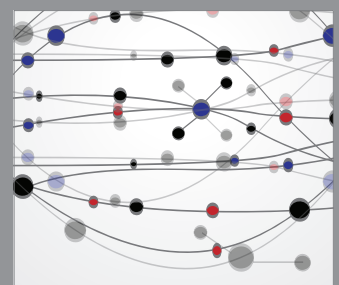

The Scientific World Journal
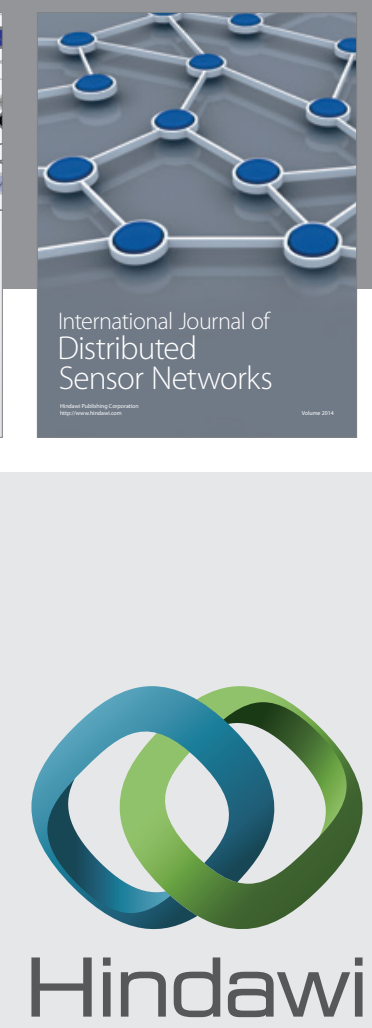

Submit your manuscripts at

http://www.hindawi.com
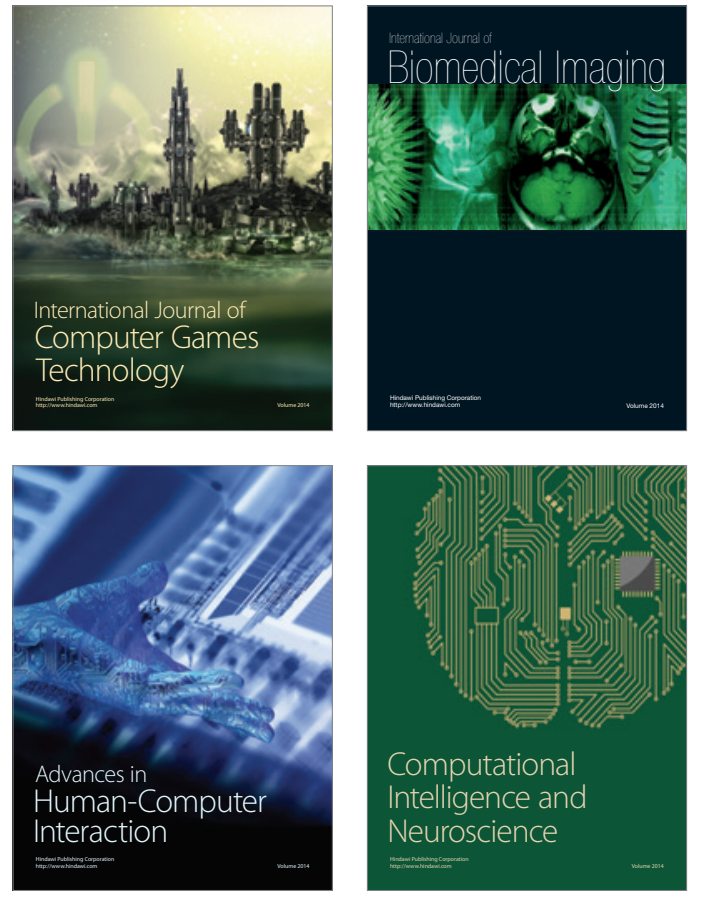
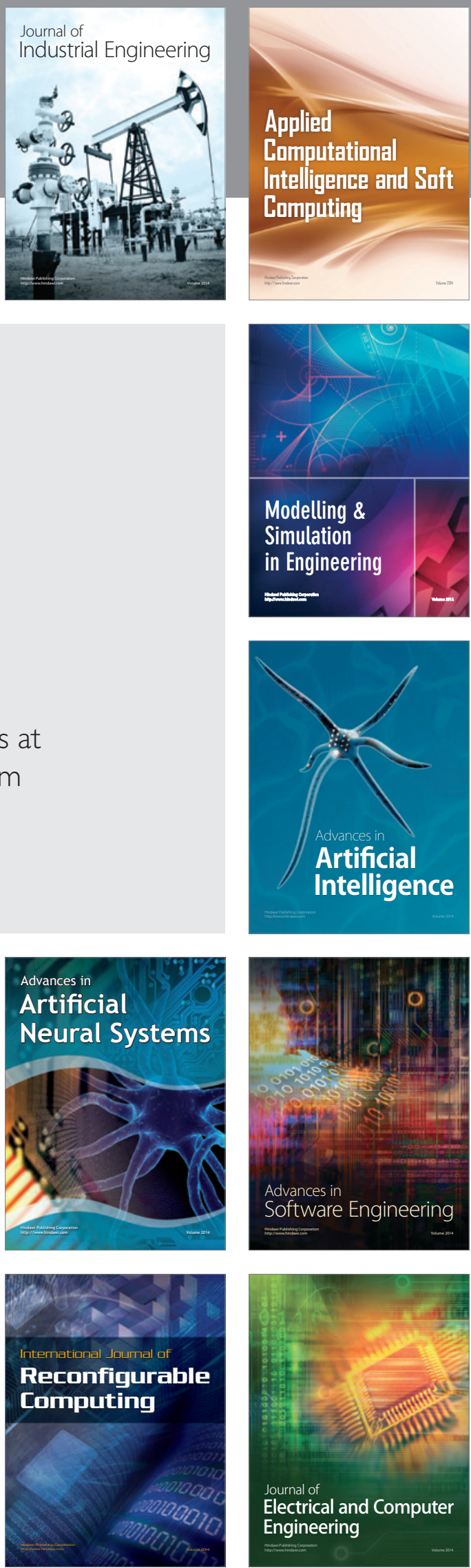